\title{
Treatment monitoring in syphilis using the autoanalyser
}

\author{
D. E. MACFARLANE AND A. MCMILLAN \\ From the City Laboratory and Department of Venereology, Glasgow
}

SUMMARY Treatment monitoring in syphilis using the automated Reiter protein complement fixation test and the automated reagin test was investigated over a two-year period. Clearly defined response patterns were demonstrated in primary and secondary syphilis and, to a lesser extent, in latent syphilis, thus facilitating the assessment of treatment and the identification of treatment failures and reinfection. No obvious treatment failures were detected in those patients receiving penicillin, but two failures were noted in a group of patients with secondary syphilis treated with doxycyline. The combination of two automated tests overcame some of the disadvantages inherent in the use of a single reagin test.

\section{Introduction}

The Technicon Autoanalyser has been used in this laboratory since 1973 to perform screening tests for syphilis. For the first two years the reporting format used with the manual tests was followed, and the results of the automated Reiter protein complement fixation test (ARPCFT) were given as positive or negative. This policy was changed in 1975, and quantitative results were reported for both the ARPCFT and the automated reagin test (ART). Clearly, monitoring antibody levels with two different serological tests gave important additional information regarding response to treatment and stage of infection. We, therefore, decided to analyse the previous two years' results from two of the local clinics.

\section{Material and methods}

The automated tests were performed according to methods previously described (MacFarlane et al., 1976).

Records were examined for a period of 24 months, and those patients with a sufficient number of antibody determinations were selected for analysis and classified as having primary, secondary, early latent (in the first two years), or late latent syphilis. Most of these patients had been treated with procaine

Address for reprints: Dr D. E. Macfarlane, The City Laboratory, 23 Montrose Street, Glasgow G1 IRN

Received for publication 22 June 1978 penicillin, 600000 IU daily for 10 days (in cases of primary syphilis, group 1) or for 14 days (in cases of secondary syphilis, group 2; and latent syphilis, groups 3 and 4). A number of patients with secondary syphilis had been treated with doxycycline, $100 \mathrm{mg}$ eight hourly for 14 days (group 5). Thus we were able to compare the two methods of treatment.

\section{Results}

GROUP 1

The serological records of 21 patients with primary syphilis are shown in Table 1 . Before treatment $83 \%$ had positive ART readings and $95 \%$ had positive ARPCFT results (Fig. 1). (For the purpose of contracting Figures 1-6 any serology result which became negative during the course of treatment was presumed to remain negative unless subsequent test results proved otherwise.) Four months later all ART results were negative, and by six months both serological tests gave negative results in all cases. Before treatment the mean ART titre was $1 / 7 \cdot 3$, and the mean level of Reiter antibody was 2036 absorbance units (au) (100 au = antibody detectable at a dilution of 1/4); these figures declined rapidly after treatment to $1 / 1.5$ and 691 respectively at eight weeks (Figs. 2 and 3). Low levels of Reiter antibody were still detectable at four and five months (Fig. 3).

\section{GROUPS 2 AND 5}

The serological records of 11 patients with secondary syphilis treated with penicillin (group 2) and 10 patients with secondary syphilis treated with doxy- 
$\underline{\underline{q}}$
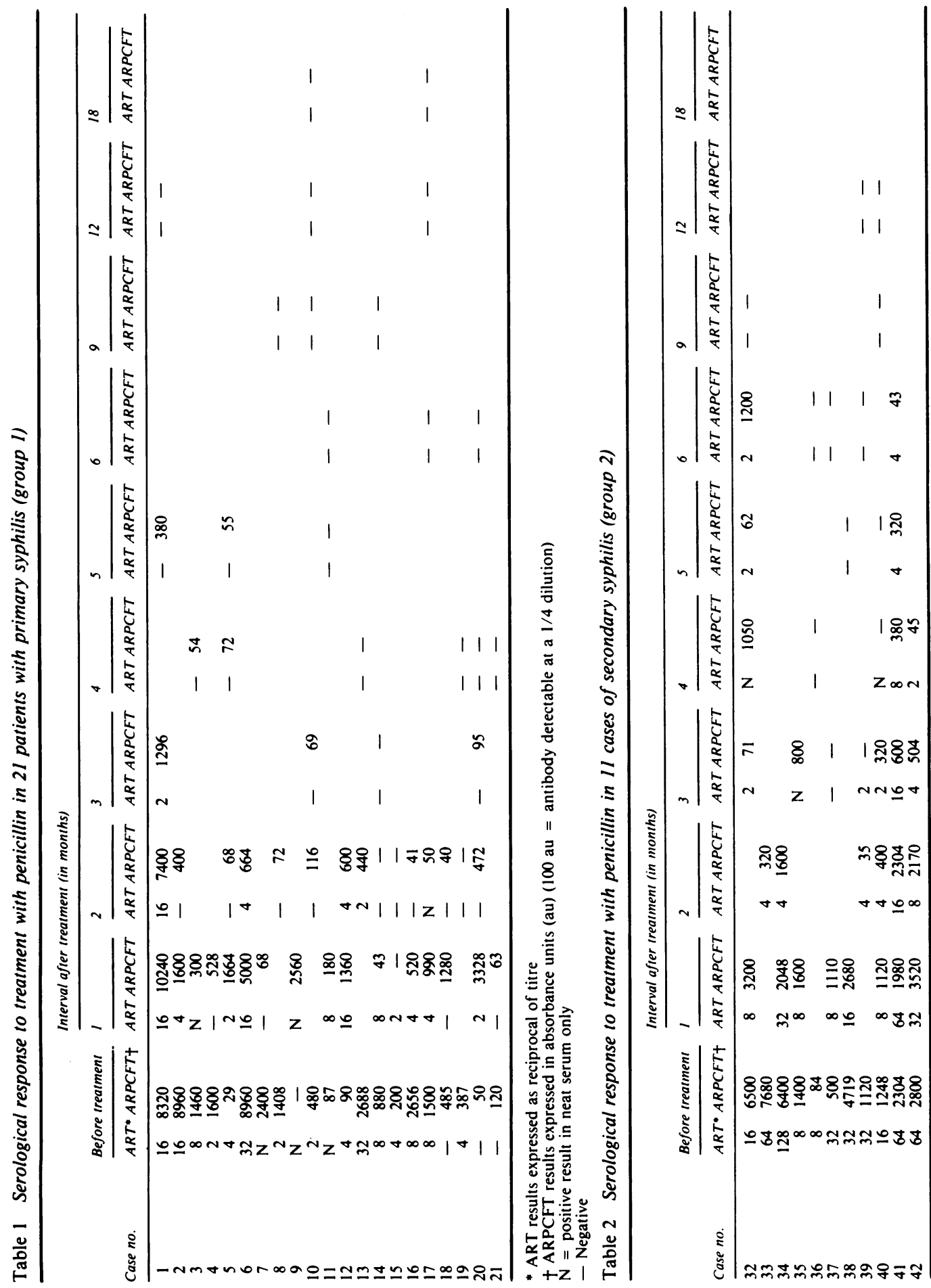

망.

$\overrightarrow{\bar{ज}}$

등

$\frac{\bar{\sigma}}{\bar{\sigma}}$

क

$\overrightarrow{0}$

$\overrightarrow{\vec{\omega}}$

$\stackrel{\infty}{\circ}$

담

$\omega$

जิ

윽

c

จิ

$\overrightarrow{0}$

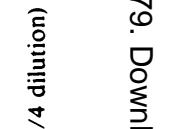




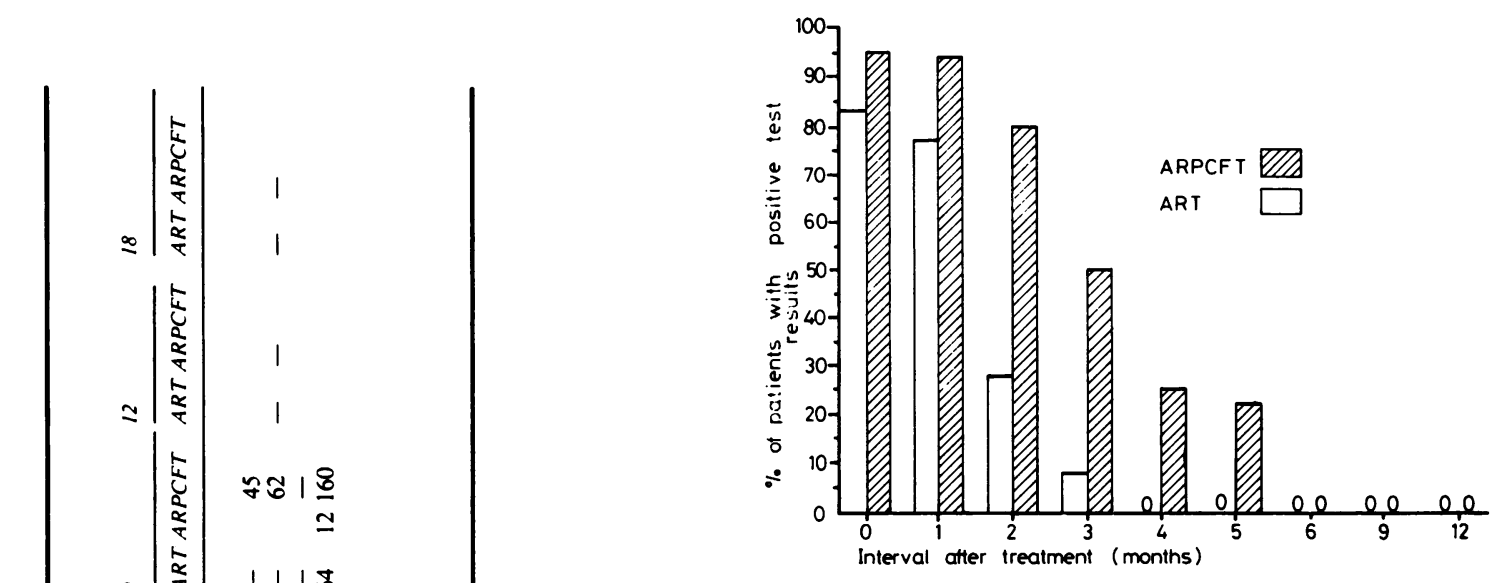

Fig. 1 Results of ARPCFT and ART for 21 patients with primary syphilis treated with penicillin (group I)

cycline (group 5) are shown in Tables 2 and 3 respectively. Both tests gave positive results in all cases before treatment. In group 2, the ART and ARPCFT results were positive for eight weeks after the start of treatment, but by six months the ART and the ARPCFT results were negative in $57 \%$ and $71 \%$ of cases respectively (Fig. 4). By nine months all results were negative. Before treatment the mean ART titre was $1 / 42 \cdot 2$ and the mean level of Reiter antibody was 3160 absorbance units (Figs. 2 and 3 ). A sharp fall in ART titre occurred in the first eight weeks after treatment followed by a more gradual decrease over the next four months (Fig. 2). The mean Reiter antibody response followed a similar pattern with a sharp fall during the first three months and a more gradual decline to zero at nine months (Fig. 3).

In the group treated with doxycycline (group 5) both tests gave positive results before and for four weeks after treatment (Fig. 5). At five months $50 \%$ of the patients had no Reiter antibody and all ART results were negative. Twelve months after treatment all serological tests gave negative results. The mean ART titre before treatment was $1 / 12$ with a corresponding figure of 2550 au for the ARPCFT. The ART titre had fallen to $1 / 1 \cdot 5$ after eight weeks and reached zero at five months (Fig. 2). There was a more gradual fall in Reiter antibody to 58 au at four months and thereafter low levels persisted until nine months (Fig. 3). Two treatment failures occurred in group 5. One patient (Case 26; Table 3) defaulted after his initial visit and was not seen for a period of six months. Serological tests at six months showed a static ART titre of $1 / 16$ and a rise in Reiter antibody from 1792 au to 6400 au (Table 3). This patient failed to return to the clinic for a further three months, by which time his ART titre had risen to 1/64 and 


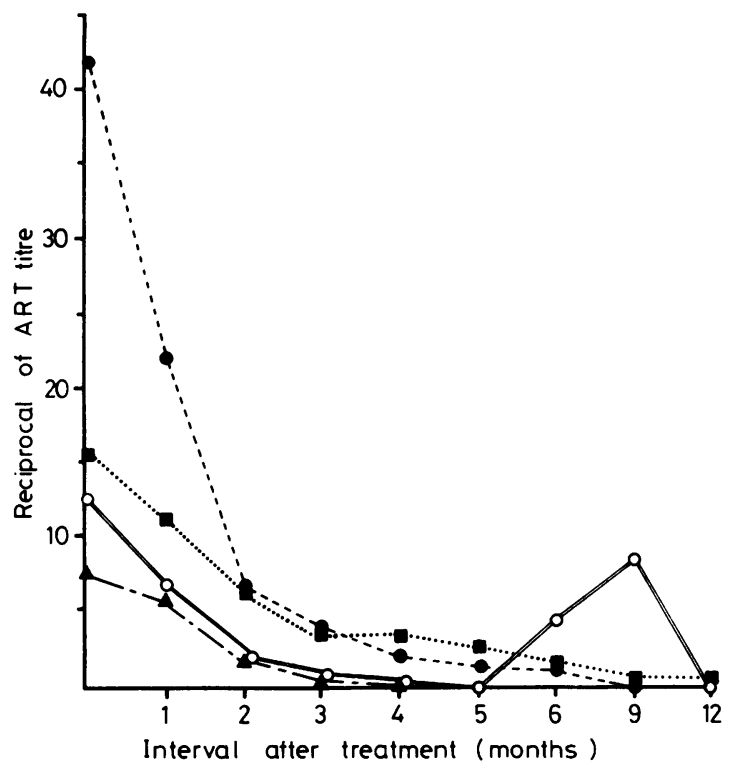

Fig. 2 Mean $A R T$ results before and after treatment in four patient groups. (The peak at nine months in group 5 represents treatment failures.)

^_- group I (primary syphilis treated with penicillin); --- group 2 (secondary syphilis treated with penicillin); -- group 3 (early latent syphilis treated with penicillin); $\bigcirc--0$ group 5 (secondary syphilis treated with doxycycline)

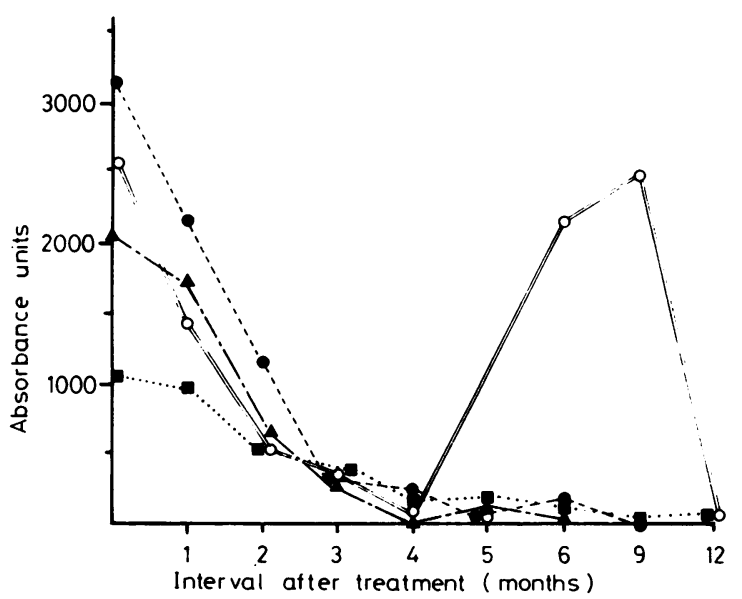

Fig. 3 Mean ARPCFT results before and after treatment in four patient groups. (The peak at nine months in group 5 represents treatment failures.) ^-_ group I (primary syphilis treated with penicillin); --- group 2 (secondary syphilis treated with penicillin); --- group 3 (early latent syphilis treated with penicillin); $0--0$ group 5 (secondary syphilis treated with doxycycline)

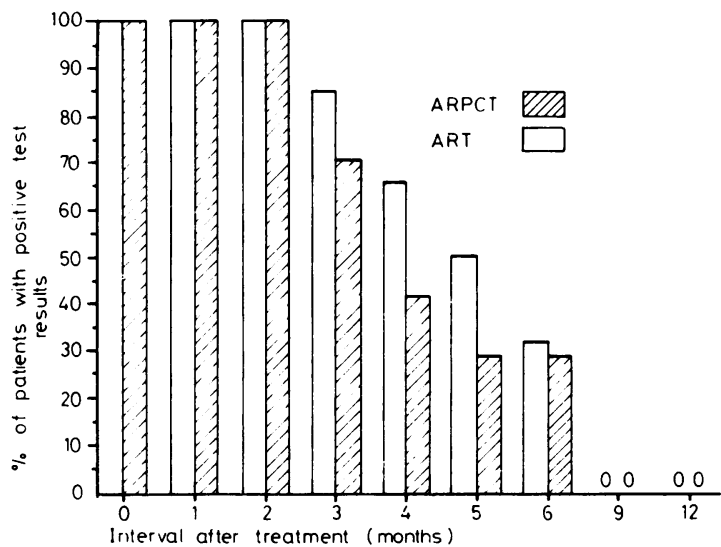

Fig. 4 Results of ARPCFT and ART for 11 patients with secondary syphilis treated with penicillin (group 2)

Reiter antibody levels to $12160 \mathrm{au}$. One patient (Case 31; Table 3) appeared to be responding adequately to treatment, the ART titre falling from $1 / 16$ to zero by three months and Reiter antibody falling from 3840 au to 53 au by four months, but by six months both antibody levels had climbed to pre-treatment levels. Both of these cases were assessed clinically as treatment failures rather than as reinfections.

\section{GROUP 3}

The serological records of eight patients with early latent syphilis (group 3) are shown in Table 4 and Fig. 6. The mean ART titre before treatment was 1/15.75 and the mean Reiter antibody level was 1079 au (Figs. 2 and 3). The antibody levels fell gradually over a period of six months and then tended to persist at low levels.

\section{GROUP 4}

Of the four patients with late latent syphilis (group 4) fluctuating antibody levels were clearly evident in three, and this makes any assessment of response to treatment very difficult (Table 4).

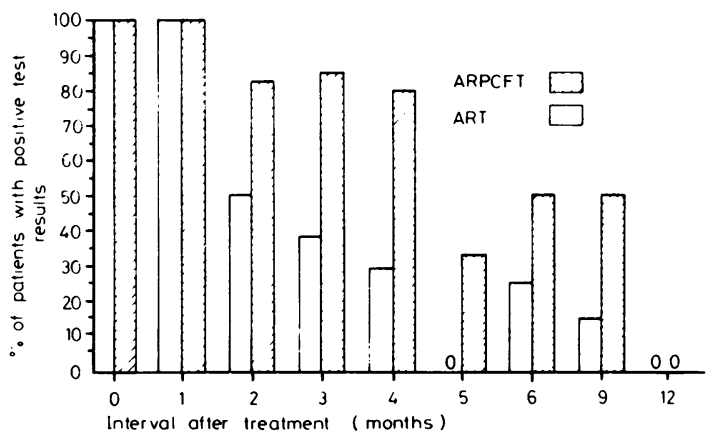

Fig. 5 Results of ARPCFT and ART for 10 patients with secondary syphilis treated with doxycycline (group 5) 


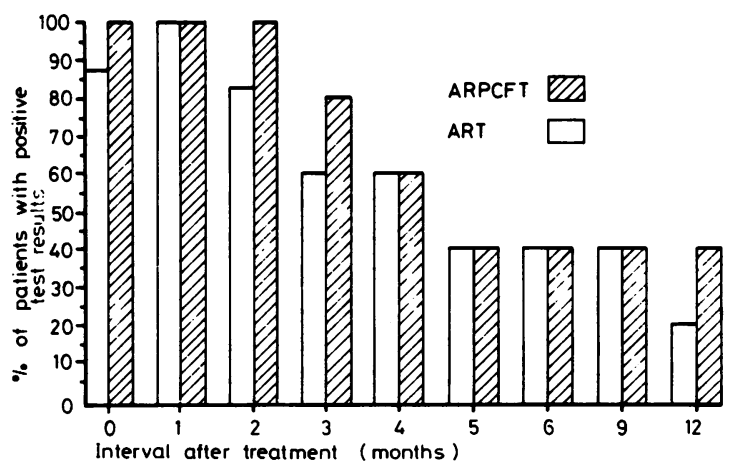

Fig. 6 Results of ARPCFT and ART in eight patients with early latent syphilis treated with penicillin (group 3)

\section{Discussion}

The diminishing antibody levels in patients with primary syphilis after treatment with procaine penicillin followed previously reported patterns (Wilkinson, 1970), the ART giving negative results by four months and the ARPCFT by six months (Fig. 1).

There were some interesting differences in the two groups of patients with secondary syphilis (groups 2 and 5). In the group treated with penicillin (group 2) the percentage of cases with positive ARPCFT results declined more rapidly than that of those with positive ART results (Fig. 4). This finding agrees with a previous observation by Schofield (1975). In the group treated with doxycycline (group 5) the position was reversed, with the positive ART results declining more rapidly (Fig.5). In both tests antibody levels in group 5 declined to zero earlier than those in group 2 (Figs. 2 and 3). These differences in response to treatment can probably be attributed to the higher initial antibody levels in patients in group 2 (Figs. 2 and 3).

The two treatment failures with doxycycline recorded in group 5 (Table 3 ) are in clear contrast to the success of penicillin in the treatment of patients with primary and secondary syphilis; those with latent syphilis are more difficult to assess. It was assumed that these treatment failures were the result of incomplete courses of treatment. It is, however, possible that treatment with doxycycline would be more liable to failure as doxycycline is bacteriostatic and not bacteriocidal.

On the basis of our results, successful treatment will give negative serological results in both tests, after three to six months in cases of primary syphilis and after three to nine months in cases of secondary syphilis. It is possible that low levels of Reiter antibody ( $<100 \mathrm{au})$ may persist for some time in a few cases, but the large number of defaulters in this 
series prevents any accurate observations on this matter.

In the patients with early latent syphilis (group 3) there was a gradual reduction in antibody levels for six months after treatment, with a tendency for low levels to persist thereafter in some cases. The Reiter antibody levels in this group before treatment were lower than those recorded in patients with primary and secondary syphilis.

Fluctuations in antibody levels are evident in three of the four patients with late latent syphilis (group 4), and there is no serological evidence of a definite response to treatment. In experimental infections in rabbits (Frazier et al., 1952) treponemes were detected at intermittent intervals in the blood in cases of latent syphilis. The fluctuating antibody levels in latent syphilis seem to provide serological evidence for a similar intermittent bacteraemia in man.

For many years treatment monitoring has consisted of measuring cardiolipin antibody levels with the Venereal Disease Research Laboratory (VDRL) slide test or its carbon antigen equivalents. Such a system has several drawbacks. The reagin test results may remain negative (Cases 18 and 21; 52 and 54) or become fleetingly positive (Cases $46,4,7,10$, and 20). The change in reagin antibody levels may be delayed or occur at insignificant levels. This can be seen in Cases 9, 14, 22, and 44. It is particularly obvious and of some importance in one of the treatment failures (Case 26). The doubling-dilution method of measuring antibody levels is so crude and liable to error that only a pronounced rise or fall can be regarded as significant.

This investigation has brought to light several advantages in using the ARPCFT to monitor treatment. The more precise measurement system gives more accurate and reliable antibody estimations. The sensitivity of the ARPCFT allows detection and monitoring of very low antibody levels at all stages of infection, including early primary syphilis where it is one of the first tests to give positive results.
The serological results in cases of untreated, early primary syphilis are not always predictable. This is best illustrated by the results of a recent group of cases of early, primary syphilis (Table 5l. In four of

Table 5 Initial serological result in six recent cases of early primary syphilis

\begin{tabular}{llll}
\hline \multicolumn{5}{c}{ Serological test results } \\
\cline { 2 - 4 } Case no. & FTA-ABS & ARPCFT+ & ART \\
\hline 55 & ++ & - & $1 / 4$ \\
56 & + I & 60 & - \\
57 & + & 120 & - \\
58 & + I & 60 & $1 / 2$ \\
59 & ++ & 63 & - \\
60 & + & 43 & - \\
\hline
\end{tabular}

*ART results expressed as reciprocal of titre

tARPCFT results expressed in absorbance units (au) (100 $\mathrm{au}=$ antibody detectable at $\mathrm{a} 1 / 4$ dilution)

\#FTA results from borderline $+/-$ to strongly positive $++\cdot++$

these cases the ART gave negative results, in one case the ARPCFT result was negative, and in two cases the FTA-ABS test result was not positive. In our experience the FTA-ABS test and the ARPCFT are the two tests whose results become positive first in cases of early syphilis closely followed by the ART. It is, however, important to realise that any permutation of these three tests may combine to give positive or negative results in cases of early primary syphilis.

Treatment monitoring using both tests tends to overcome any disadvantages inherent in the use of a single reagin test and acts as a dual checking system on all results.

\section{References}

Frazier, C. M., Bensel, A., and Keuper, C. S. (1952). American Journal of Siphilis, 36, 167.

Macfarlane, D. E., Hare, K., and Elias-Jones, T. F. (1976). Evaluation of large-scale screening tests for syphilis. Journal of Clinical Pathologv, 29, 317-321.

Schofield,C. B.S. (1975). Sexually Transmitted Diseases, 2ndedition p 71. Churchill Livingstone: Edinburgh.

W'ilkinson A. E. (1970). The positive Wasserman reaction: investigation and interpretation. British Journal of Hospital Medicine. 4(1), 47-54 